\title{
Das Bild der Natur bei Zwingli
}

\author{
von FrITZ BüsSER
}

Wenn wir am heutigen Abend, am Todestag unsres Zürcher Reformators Huldrych Zwingli einmal über das Bild der Natur bei Zwingli reden, geschieht das vornehmlich aus zwei Überlegungen heraus: einmal weil die Beschäftigung mit der Natur für uns protestantische Menschen an sich eher etwas Seltenes ist, und dann, weil heute, im Zusammenhang mit dem Fortschritt der Technik überhaupt ein zunehmendes Fortschreiten des Menschen auch von der Natur festzustellen ist. Sicher gibt sich der moderne Mensch mit der Natur ab; aber diese Beschäftigung ist einseitig technisch-wissenschaftlich orientiert: er erforscht den Mechanismus der Natur, er macht sich die Kräfte der Natur untertan, um dann die Natur zu beherrschen, auszubeuten. Dieser moderne Mensch denkt aber kaum mehr daran, daß es neben dieser technisch-naturwissenschaftlichen Betrachtung auch noch eine ästhetische, religiöse Naturbetrachtung gibt: neben dem Mechanismus, neben den Gesetzen und Ordnungen der Natur auch noch das, was ich einmal die Schönheit und Würde, die Mächtigkeit und Erhabenheit der Natur nennen möchte, neben der Bemeisterung und Unterwerfung, zum Teil sogar Gefährdung und Ausbeutung der Natur die stille Ehrfurcht, das Ergriffensein des Menschen von der Natur als der Schöpfung, als dem Werk Gottes. Daß diese religiöse Naturbeziehung übersehen, vernachlässigt wird, ist nun allerdings nicht bloß eine Folge unsrer allgemein einseitig orientierten Gegenwart, sondern leider auch die Folge einer etwas einseitigen Theologie. Wie bei uns vor allem Fritz Blanke nicht müde wird zu sagen und zuletzt in einem aufrüttelnden Aufsatz über «Unsere Verantwortlichkeit gegenüber der Schöpfung» in der Festschrift für Emil Brunner dargelegt hat, sind gerade für den protestantischen Menschen Natur und Gott gerne geschieden. "Das sittliche und das kosmische Leben stehen ohne Beziehung nebeneinander, die Frömmigkeit ist innerlich, jenseitig», sie ist streng anthropozentrisch, das heißt auf den Menschen bezogen auch in der Lehre von der Schöpfung. Die Theologen hüten sich auch nur vor einem Anflug sogenannter natürlicher Theologie, obschon doch selbst die Bibel - in den Naturpsalmen 8, 19, 29, 104 und 148 etwa, in Rö. 1, 20 u.a. - die Natur als ein Spiegelbild von Gottes Weisheit, Kraft, Ehre, von Gottes Größe behandelt, obschon für tief religiöse Menschen, wie einen Franz von Assisi, schon im grauen Mittelalter nicht bloß der Mensch, sondern die ganze Kreatur Gottes Güte preist und auch nicht bloß der Mensch, sondern die gesamte Kreatur 
Gegenstand der in Christus geleisteten Erlösung ist nach den Worten Christi: "Siehe ich mache alles neu» (Apk. 21, 5), nach den Worten des Paulus an die Kolosser $(1,19)$, Gott habe durch Christus alle Geschöpfe im Himmel und auf Erden mit sich versöhnt; an die Epheser, der Wille Gottes, der sich in der Fülle der Zeiten erfüllen werde, gehe dahin, da $B$ «aller wieder... unter Christus vereinigt werde, was auf Erden und im Himmel ist» (Eph. 1, 10).

Wir wollen heute abend nun, wie gesagt, uns einmal fragen, welches Bild Zwingli sich von der Natur gemacht hat. Wir wollen dabei den Reformator selber ausgiebig zu Wort kommen lassen und beginnen, etwas äußerlich, mit der Feststellung, daß Zwingli jedenfalls ein lebhaftes Naturempfinden sein eigen nennen konnte und daß er sich in Dingen der Natur erstaunlich gut ausgekannt hat. Wie vor allem Walther Köhler und Oskar Farner literarisch gezeigt haben, hat Zwingli in seine theologischen und politischen Schriften ziemlich viel interessantes naturkundliches, naturwissenschaftliches, auch medizinisches Material einfließen lassen ${ }^{1}$; natürlich weiß man seit alters auch, daß $Z$ wingli in seiner Schrift «Über die Vorsehung» sogar so etwas wie eine eigene Weltanschauung gezimmert hat, wie Walther Köhler sagt: eine «eigentlich naturwissenschaftliche Broschüre auf deterministischer Grundlage», in der er «die göttliche Vorsehung auch in der natürlichen Zweckmäßigkeit zu erfassen und die Ähnlichkeit zwischen Vorgängen in der Natur und in der Geisteswelt zum Naturgesetz in der Geisteswelt ahnend verknüpfte». Leider fehlt uns die Zeit, jetzt näher auf die verschiedenen Wurzeln dieser Naturbetrachtung einzugehen; für unsere Zwecke mag zu wissen genügen, daß sie genährt ist aus der Bibel, aus seiner gründlichen Lektüre des Aristoteles, der Stoa, des Plinius, der beiden Picus della Mirandola, des Galen, aus dem Verkehr mit Naturwissenschaftern und Philosophen seiner Zeit, nicht zuletzt aber aus eigenster persönlicher Erfahrung, aus eigenen Erlebnissen. Uns interessiert jetzt vielmehr, was Zwingli mit seinem Blick für die Natur, mit der Kraft seiner Anschauung, mit seinen literarischen Kenntnissen über die Natur angefangen hat.

Da ist das erste sicher dies, daß Naturerkenntnis und Naturempfinden

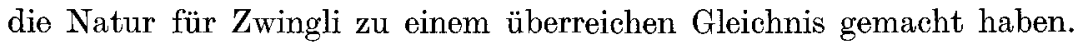

1 Oskar Farner, Huldrych Zwingli, Bd. 1, bes. S. $107 \mathrm{ff.}$; $132 \mathrm{ff.;}$ Walther Köhler, Medizinisches bei Zwingli. Vierteljahresschrift der Naturforschenden Gesellschaft in Zürich, Bd. LXXXII, 1937, S. 457 ff.; Walther Köhler, Zwingli, S. 10f. (Die Schweiz im deutschen Geistesleben 9). Leipzig 1923. - Ich verweise hier dankbar auch auf einen Aufsatz von Heinrich Bornkamm über Luthers Stellung zur Natur, dem ich manche Anregung verdanke (erschienen in: Luthers geistige Welt, Gütersloh 1953, S. 199 ff.). 
Genau wie Luther wußte ja auch Zwingli um die Zweckmäßigkeit guter Bilder. Wie er dementsprechend mit allerlei historisch-literarischen oder militärischen Bildern Dinge des öffentlichen Lebens veranschaulicht hat, so versuchte er auch immer wieder, politische, menschliche, kirchliche und theologische Dinge seinen Lesern und Zuhörern durch Gleichnisse aus der Natur verständlich und anschaulich zu machen. Ich erinnere, da es sich da wohl um allgemein bekannte Dinge handelt, nur kurz an ein paar politische Gleichnisse : etwa daran, wie Zwingli in seinem Fabelgedicht vom Ochsen, 1510, schon das Schweizervolk mit einem «Muni » vergleicht (Z I, S. 11, z. 5ff.), wie er bei anderer Gelegenheit die beiden Hauptorte der damaligen Eidgenossenschaft, Bern und Zürich sieht «als zween Ochsen vor dem Wagen, die an eim Joch ziehen" (zit. nach Farner I, S. 110); oder meinetwegen auch an den häufig aufmarschierenden «mit Gold und Syden überhänckten Mulesel» des Papstes und seiner Würdenträger (Z IV, S. 107; V, S. 440; V, S. 48). Ich erinnere auch nur kurz an die Vergleiche, die Zwingli Luther anhängt: da dieser einmal nach dem Knoblauch und $Z_{\text {wiebeln in }}$ Agypten schmeckt (weil er seine katholische Denkart noch nicht völlig lassen kann) (S II, Abt.2, S. 120), oder da dieser (unbelehrbar) sich winden und wenden soll wie der Häher im Kleb (an der Leimrute): «ie meer derselb schryet, gwägget und zablet, je mee er klebt» (S II/2, S. 221). Interessanter, weniger zeitgebunden, auch für uns treffend, gültig scheinen mir folgende Gleichnisse Zwinglis für uns Menschen, für unsere Art überhaupt. Da kann er etwa, alles umfassend über die Erkenntnis des Menschen schreiben: "Den Menschen erkennen ist so mühsam wie einen Tintenfisch fangen; denn wie dieser sich in seinem schwarzen Saft verbirgt, damit man ihn nicht ergreifen kann, so entwickelt der Mensch, sobald er merkt, daß man ihn will, plötzlich so dichten heuchlerischen Wolkendunst, daß auch das schärfste Auge ihn nicht fassen kann» (Z III, S. 654; auch Anm. 2). Oder über das Leben des Menschen: «Also wil uns Got üben; wie ein Esel (sind wir), der ein Burdi durch einen wüsten Weg treit; der muß nit fallen, nit stillstan, immerdar fürgan; fallt er, wider uffstan» (S VI, S. 437). Oder dann über unsere Sündhaftigkeit gleich zwei Vergleiche: «Unser Fleisch ist eim Mulesel glich; der lugt (schaut, lauert), wie er sich usrissen mög und entlauffen» (S IV, S. 441), und die Konstanz der Art betonend: Wolf bleibt Wolf. «Der jung wolff, diewyl er noch blind ist, weißt er nütz vonn schaffzwacken; noch so ist die ard (diese Anlage) in imm. Sobald er aber erwachst, so hebt er denn an ärdelen» (Z IV, S. 308f.); genau gleich steckt im Menschen schon von Anfang an die Erbsünde als Bresten, sie aktualisiert sich aber erst später zur persönlichen Schuld. Interessant ist in diesem Zusammen- 
hang übrigens auch eine Stelle aus der Einleitung zur Auslegung des Propheten Jeremia: «Ein Wolf oder sonst ein Tier wird nur durch Schläge erzogen. So wird das Fleisch durch die Obrigkeit zahm gemacht. Die Hinterlisten stecken fortwährend im Tier; sobald es keine Schläge mehr befürchten muß, ist es wieder seiner angeborenen Eigenart preisgegeben. So ist das Fleisch nur durch die Furcht vor den Gesetzen und dem Schwert im Zaume zu halten; es muß drum ständig einen Bändiger haben ${ }^{2}$. Ich übergehe jene Stellen, die in der gleichen Vorrede - biblischen Gleichnissen entsprechend - vom Ausreißen des Unkrautes und der Disteln (1.c. S 169), von der Pflege des Gartens überhaupt handeln, um an einem weitern Beispiel noch den Menschen eher in seinen positiven Eigenschaften zu malen: "Wir aber (wo man uns fraget) wurdend sagen, der Mensch wäre under allen Gschöpfften das aller sältzamest und wunderbarlichest; dann er ouch die engelische Schöne wyt übertrifft, glych als so man einen Hanen neben einem Stier beschouwte, so funde man, daß der Stier vil der gwaltiger und stercker wäre. Wenn man aber des Hanen künigklich Zierd. ermißt, syn groß, herrlich Gemüt, synen stoltzen Gang, syn flyßige Nachtwacht, die niena fält, die schönen Künst, die einem Houptman und Fůrer zůgehörend (ob der glych nun einer Chütt (Schar) Hennen fürgesetzt ist), wär möchte doch sich syn gnůg verwunderen? Also ist der Engel ein hoche, edle Substanz, ein reiner, luterer Geyst; wenn du aber den Menschen darneben ermissest, der nit allein ein himelisch, sunder ouch damit ein yrdisch Ding ist, wie kan es syn, daß du nit gar in Verwundernuß verzuckt werdest und erstunest? » (Zwingli : Hauptschriften, Bd. 2 = $\mathrm{ZH} \mathrm{2,} \mathrm{S.} \mathrm{131.)}$

Mit dem Federvieh kommen wir schließlich auch aufs kirchlichtheologische Gebiet. Da brüten die Sektierer ihre Eier den Hühnern gleich in Schlupfwinkeln (Z IX, S. 112), da schnattern die Täufer wie Gänse ( $Z$ IV , 587). Für die Gegner $Z$ winglis erscheint dann einmal aber auch das Bild gefährlicher Wildwasser, die große Verheerungen anrichten: «Das nimpt gäch alles das hyn, das es erlangt, und mert sin krafft darmit. Es werdend zum ersten nur kleine steinle bewegt; dieselben bewegen darnach mit offt anpütschen die größeren, bis das die rüfe so groß und mechtig wirt, daß sy alles, so iro entgegen stat, uffrumet und hynnimpt, und hinder iro nüts laßt denn ein unnützen rüwen (wertloses Leid), klag und entschöpffung der schönen jucharten... Glych also thủt eigenrichtigkeit und zank" (Z IV, S. 215). Im allgemeinen dienten

${ }^{2}$ Aus Zwinglis Predigten zu Jesaia und Jeremia, herausgegeben von Oskar Farner, Zürich 1957, (zitiert: Predigten), S. 159. 
allerdings gerade diese elementaren Gewalten $Z$ wingli eher als Gleichnisse für Gott: so entspricht dem Föhn einmal der hl. Geist: "Wo der geist Gottes kuchet (haucht, weht), da verwäyet er alles gstüpp (Stoppeln) und güsel (Spreu) der glychsnery und truckt andere blůst (Blüten) herfür» (Z IV, S. 206); dem Rhein die Gewalt von Gottes Wort: «Tủnd umb Gots willen sinem wort gheinen drang an! Dann warlich, warlich, es wirt als gwüß (so sicher) sinen gang haben als der Ryn, den mag man ein zyt wol schwellen, aber nit gstellen » (Z III, S. 488). Schließlich spürt Zwingli in den Bildern der Natur Gott selber. So gibt er zum Beispiel für Gottes Allgegenwart das schöne Beispiel der Sonne: «Die Sonn wirdt von allen Menschen in der gantzen Welt gesehen, erlüchtet alle Welt, erfrüchtiget (befruchtet) und wermt alle Ding zủ eyner Zyt, und nüßt die gantzen Sonnen und weydet das kleynest Gräßli glich als wol als der größte Berg ald Boum. Noch ist der Dingen keins, das darumb allenthalb sye, da die Sonn ist, ja es begert keins, by iren zů sin oder iren Gang zu thưn, sonder es benůgt sich, daß es von iren geheytzt und läbend gemacht wirdt. Und sind doch alle Ding under der Sonnen, läbend in ihr, weydend sy, sehend sy nit zum Teyl, sonder gantz. Also durchgadt Gott alle Ding, ist allernthalb, erfröuwt, macht läbendig alle Ding, wirdt von allen Dingen genossen, genutzet und geweydet, ouch von den Unglöubigen, die es glich nit wüssend... Deßhalb die Ußerwelten Gott so gentzlich sehend als wir die Sonnen, und so genůgsam.» (ZH 2, 41f.) ${ }^{3}$.

So waren, um es bei diesen Beispielen bewenden zu lassen, Haus und Wald, Garten und Berge, Tiere und Pflanzen, die Elemente, selbst die Sonne für Zwingli einmal voller Anschauung für das, was Gott und die Menschen sind, was Gott für die Menschen tut. Zwingli hat sich dabei nun allerdings nicht allein auf die Freiheit der dichterischen Phantasie,

${ }^{3}$ In diesem Zusammenhang ist an eine weitere interessante Stelle in der Predigt "Über die Vorsehung» zu erinnern: «Dann alle Thier, die da sind, die sind eintweders zam und gschlacht, oder wild und grusam. Das Tygerthier und Crocodil kündend eben als wol die Majestat, Herrlikeit und Macht Gottes uB, als ein Ochs und ein Schaaff. Dann wie der Ochs und das Schaaff die Gủte Gottes lobend und prysend, also lobend und prysend die grusamen Thier sin Gerechtikeit, nit daß sy ir Art und Natur halb etwas thůgind, das der Gerechtikeit gemäß und änlich sye, sunder daß sich alles das, das sy thůnd, uff Unbill und fräflen Gwalt zücht... So nun die grusamen, wilden Thier mit irer Art und Natur die Ungerechtigkeit für Ougen stellend, brysend sy Gottes Gerechtigkeyt (in dem sy die Ungerechtigkeyt, on die das Angsicht der Gerechtigkeyt num nit kent mag werden, für Ougen stellend) nit minder dann die hilflichen Thier (als Kủe, Esel, Roß) syne Gưte ußkündend und prysend, daß also alle Thier (die in zweyerlei geteylt sind: schädlich und unschädlich) glychlich die Gerechtigkeyt und Güte Gottes ußkündend und prysend.» (ZH 2, S. $161 \mathrm{f}$.) 
auf die Zweckmäßigkeit der zuletzt ja durch das Wort Gottes selber angeregten plastischen Bilder berufen; wenn er die Natur voller Gleichnisse gesehen hat, glaubte er dazu (wenn ich einmal so sagen darf) auch so etwas wie ein tieferes Recht zu besitzen. Wie gerade die zuletzt zitierten Gleichnisse gezeigt haben sollten, war die Natur für Zwingli auch ein Zeichen: ein Zeichen für Gottes Weisheit oder wie Zwingli mit Vorliebe sagte, ein Zeichen für Gottes Vorsehung, damit gleichbedeutend für Gottes Gnade und Liebe.

Wir kommen damit zum zweiten Teil unsres Vortrages, der von Zwinglis Grundanschauung über das natürliche Leben handeln soll. Wir werden auch da weniger Bekanntes einfach wiederholen, als versuchen, eben ein paar, nicht zuletzt auch heute noch gültige Grundgedanken herauszuarbeiten. Zwingli war einmal bei allen naturwissenschaftlichen Kenntnissen kein Naturforscher. Er besticht zwar - wir wir schon hörten und wie wir noch sehen werden - oft durch vorzügliche Einzelbeobachtungen. Seine botanischen, zoologischen, medizinischen, überhaupt naturkundlichen Kenntnisse gehen sehr weit, er gibt auf Grund seiner medizinischen auch Gynäkologie, Psychiatrie und Otiatrie umfassenden Einsichten oft weise und praktische Ratschläge - so verlangt er etwa in seinem «Lehrbüchlein» von 1523 Mäßigkeit im Genuß von Alkohol und Delikatessen, oder er mahnt einmal seinen jungen Freund Jakob Ceporinus, mehr auf seine Gesundheit bedacht zu sein und nach Tisch 3 Stunden lang nichts Schweres und Problematisches zu lesen: «es müssen die jungen Leute für ihre Gesundheit sorgen und dürfen nicht auf die hören, die mit der göttlichen Vorsehung Schindluder treiben» (Z IV, S. 874f.).

Auch mag er durch originelle Ideen noch heute Interesse zu wecken: ich denke da etwa an seine Elementenlehre (ZH 2, S. $87 \mathrm{f}$.) oder an seine Umdeutung der altpythagoräischen Seelenwanderungslehre (ZH 2, S. $107 \mathrm{ff}$.$) .$

Das alles, solch vereinzeltes Suchen, Forschen war aber kaum mehr als ein Sammeln, wie denn bei Zwingli auch noch kaum etwas zu spüren ist von der Revolution der Naturwissenschaften von Cues über Paracelsus, Kopernikus bis zu Bruno, Kepler und Galilei, deren Zeitgenosse er zum Teil immerhin war. Im Vordergrund seiner Beschäftigung mit der Natur stand die Natur selber; Zwingli interessierte sich für die Natürlichkeit der Natur: für die Frage, was diese Natur überhaupt ist. Mit andern Worten: er interessierte sich nicht für die Vorder-, sondern für die Hintergründe der Natur. Diese Art «Naturforschung», Naturkenntnis führte Zwingli zunächst zu einem großen, ehrlichen Staunen. Der schon erwähnte Psalm 104 muß für ihn dabei so etwas wie ein Muster gewesen sein; um all die Wunder 
der Schöpfung zu erkennen, sagt Zwingli nämlich im «Commentarius über die wahre und falsche Religion» (ZH 9, S. 33) "malt dieser beides (Gottes Weisheit und Vorsehung) dermaßen ab, daß du zu sehen glaubst, wie Gott als Schöpfer die Berge wägt mit starker Hand und jeden an seinen Ort setzt, wie er die Täler mitten durchzieht und in den Tälern die kühlen Flüsse, wie er die Felder ausdehnt, wie er das wütende Meer in seine Abgründe zurückstößt, damit es durch seinen Ausbruch nicht Verwirrung stifte...» Ja, in der gleichen Schrift etwas weiter vorn weist Zwingli selber auf die Wunder der Schöpfung hin, wenn er, 1.Mose 1, auslegend sagt: «Schau, wie hier das rauhe Erdreich auf das erste Geheiß seines Schöpfers ein fröhliches Gesicht annahm! Denn als die Wasser sich verlaufen und in ihren Vertiefungen gesammelt hatten, und die Erde allein für sich dalag, bot sie einen nackten und abscheulichen Anblick. Nun wollte Gott nicht, daß sie ihre Blöße den Augen der Sterblichen zeige und befahl, daß sie sich eiligst mit grünendem Kraut bekleide und Bäume hervorbringe, um sich mit diesen zu zieren, damit sie den gleich darnach entstehenden Tieren eine Herberge, einem jeden nach seiner Art, bieten könnte. Damit nicht genug, hat er, auf daß niemals an Nahrung Mangel herrsche, dem Kraut und den Bäumen die Kraft, Samen hervorzubringen, eingepflanzt, damit, wenn sie uns alles für den Winter Notwendige dargereicht hätten, sie sich im Frühling von neuem zum selben Werke rüsten könnten, und das in unaufhörlichem Wechsel. So wir solches Jahr für Jahr in unaufhörlichem Fortgang geschehen sehen, erkennen wir da nicht die unermeßliche Kraft und Vorsehung des Schöpfers und seine der Schöpfung zugewandte Sorge und Gnade? " (ZH 9, S. 29.) Diese Sorge und Gnade erkannte Zwingli in der Speisung der jungen Raben (Lk. 12, 24; Hi. 38, 41; Psalm 147, 9), an die er mehr als einmal erinnert; er erkannte sie natürlich in den Lilien auf dem Feldet. Er erkannte sie aber auch schon in der Betrachtung einer einzigen Ranke des Weinstockes: «Sie hat einen Sproß, der durch die Mitte bis zur äußersten Spitze durchläuft. Von diesem Sproß zweigen sich achselartig (Gefäßbündel) wie Schlagadern und Hauptvenen ab; aus diesen erstrecken sich wie die (menschlichen) Eingeweide-

4 «Du hast ein RoB, eine Kuh, für die bist du besorgt; du verschaffst und kaufst das Heu, das Futter. Nun ist dir das Roß, das Vieh ja wichtiger als das Futter; um des Viehs willen verschaffst du dir das Futter. Wäre dem nicht so, so würdest du dem Futter nichts nachfragen. Kannst du mir nun sagen: warum denkst du nicht: Schau, Gott ist dein Vater und hat dich geschaffen; du bist also wertvoller als das Gras und die Früchte, die aus der Erde wachsen? Doch um des Menschen willen! Wie konnten wir denn meinen, Gott lasse das Gras und das Korn wachsen und trage keine Sorge um uns und bemühe sich nicht um uns? Das tun die Hochmütigen aber nicht, und sie vermögen doch nicht ein Gräslein anders zu machen. 
blutgefäße Ranken bis ganz zu äußerst, die den Saft richtig verteilen. Kein anderes Bild ergibt sich, wenn du den ganzen Menschen oder die ganze Welt forschend betrachtest. Und bei einem so kleinen Blatt mußt du dieses Studium aufgeben, bevor du alles begriffen hast! Sieh, wie alle Menschenweisheit auf Nichts begründet ist und wie sie gezwungen ist, ihre völlige Unwissenheit einzugestehen » (ZH 1, S. 32f.). Das Wunder der Schöpfung schildert Zwingli schließlich besonders schön am Menschen im Zusammenhang mit der ganzen Kreatur! Mit den gleich hellen Augen des Glaubens wie im Commentarius heißt es in der Schrift «über die Vorsehung »: «Der Mensch ist der Herr und Hußwirt der Welt; dann alles, das wir sehend, ist umb sinetwillen und im zů Gůtem gemacht. So nimm yetz denselbigen Dingen den Menschen hin, hast du nit alle Ding beroubt und als vil als zů Witwen gemacht? Wäm wirt die Sonn schynen? Wän wirt sy wermen? Wäm wirdt der fruchtbringend West wäyen? den Thieren? Wär wirdt die Thier nutzen und nießen? Wäm wirt die Erden offen ston, so nieman syn wirt, der verstadt, wie man alle Ding bruchen und nutzen sol ? Der aber, der so vil und großer Dingen Herr und Man ist, der můßt von notwägen ouch etwas an im haben deren Dingen, denen er fürgesetzt was; ... Deßhalb hatt Gott einen Lyb geben dem, der über alle lyplichen Ding herrschen sölte; darüber hatt er in begaabet mit einem verständigen Gemůt... Also ist der Mensch $\mathrm{u} B$ zweyen Dingen, die stracks wider einander sind, gemacht und zemen gesetzt. Dann was ist Frömbders und Verrers (Entfernteres) von der Klarheit und Liecht des Gemūts und Verstands weder die Gröby und Unbefintliche der Erden und des Lybs? Sich aber, wie der höchste Werckmeister, Gott, do er das edel Gmůt in den Kaat stoßen wolt, vorhin sölichen Kaat gebeytzt, gebeert (bearbeitet) und gstaltet habe, damit er ein sölichen eerlichen Gast mit einer Herberg, die im ungemäß wäre, nit entuneerete (verunehrte). Der Löw, do der gschaffen, gieng herfür ruch und zottecht von Haar an syner Brust, mit scharpffen Zänen, mit grusamen Klaawen. Der Bär kroch harfür ruch gehaaret an allem Lyb, mit starcken Datzen. Der Hyrtz sprang herfür mit sinem vilzinkigen Gehürn, doch haarecht, syne Fůß mit herten Klaawen ver-

Gott mißfällt nichts mehr als der Hochmut. Ferner kann vor Gott niemand bestehen. Diese zwei Dinge sollten uns den Hochmut zu Boden schlagen; denn wenn Gott mit seinem Geist gegenwärtig erscheint, so fällt alles zu Boden, wie vom Wind die Blumen auf der Heide. So auch David in der Rolle Gottes: Ich sah den Gottlosen hoch erhaben wie die Zedern des Libanon. Prüfen wir uns also! Je näher wir dem Hochmut sind, desto näher sind wir dem Verderben. » Aus Zwinglis Predigten zu Jesaja und Jeremia, hgg. von Oskar Farner, Zürich 1957 (zitiert Zwingli, Predigten), S. 71. 
sorgt, und alle andren Thier, ein yetlichs was mit synem Spieß und Schilt gewappnet. Aber der menschlich Lyb (ach Gott!), wie hat er doch so gar kein Rühe noch Scherphe niena! Wöliches etlich der Arbeytsäligkeit (Mühsal) zůschrybend; ich aber vermeyn, es sye ein sunderbar Kleynot und glücklich Zeychen. Dann diewyl der Mensch darzů geschaffen was, daß er Gott und alle Creaturen nießen sölte, hat er sich wol gezimpt, daß er einen Lyb hette, der der Senfftmůtigkeyt, dem Friden und der Früntschafft füglich und bequäm wäre. Deßhalb hatt das Kaat, daruß der Lyb des Menschen gemacht ist, flyßiger můßen gebeitzt und gebeert werden... Deßhalb dann die Herberg und daß Huß des himmelischen Gemůts allenthalb glimpffig, glatt und lind ist. Die Thier, die fallend nider uff vier Fůß; der Mensch aber staat übersich ufgerichtet uff zweyen, daß er dester strackter und ufrächter den Himmel, den er verhofft zů erben, anschouwen möge. Was sol man vil sagen? Die schöne und liebliche Gstalt des menschlichen Lybs (so man sy gegen andren läbenden Dingen verglychet) ist unussprächenlich.» (ZH 2, $132 \mathrm{ff}$.) $\mathrm{Muß}$ ich noch erwähnen, wie Zwingli auch die Ordnungen der Natur bewundert, wie er (wieder in der Predigt von der Vorsehung: ZH 2, S. 139) etwa «die gefläcketen Parden», die «grymmen Tygertier», die «behilflichen Ochsen», die «klugen und wolmerckenden Roß, die röubigen Adler, die wolriechenden Gyren », die «frölichen Nachtgallen », auch die "lutschryenden Amßlen», Thunfische, Delphine, Schnecken und Polypen... erwähnt? Oder muß ich noch jene wunderbare Stelle zitieren, da Zwingli nach Apostelgeschichte 17 sagt: "Es ist ouch nit alleyn der Mensch göttlichs Geschlächts, sunder ouch alle Creaturen; wiewol eine mee edler und thürer ist weder die ander, so sind sy doch des Ursprungs und Geschlächts halb alle uß Gott und in Gott; so vil aber ein yettliche edler ist und höher, so vil mee kündet sy $u ß$ die göttliche Eer und göttliche Macht.» Und dann zeigt Zwingli diese Ehre an den Mäusen, an Igel und Murmeltier, an der Erde, an den Bergen: «Ja im Gschlächt der Müsen (die doch by uns die verachtesten sind) findt man die, die Wyßheit und Fürsichtigkeit Gottes ußkündend, so der Ygel so artlich und kunstlich an sinem Burst so vil Ops sich walend in syn Hüly yntreyt; so das Murmeltier eynen Wächter in die höhe stelt, domit die anderen, die hin und har schweyffend und der Arbeyt obligend, nit unfürsähenlich mit etwas Schräcken und Forcht überfallen wärdind; dann er mäldet alle Ding by Zyten mit synem Geschrey; darzwüschen lůgend die anderen, wo sy gůt, lind Höw findind, das nemmend sy; diewyl sy aber keynen Wagen habend, machend sy sich selbs zů einem Wagen, namlich also: das ein under inen leyt sich an Rucken, die anderen legend im alles Höw uff den Buch; das faßt er dann 
und hebt's zůsammen mit den Fůßen; denn so nimpt eins den Wagen bym Schwantz und fart also mit dem Höw in die Hüle, damit sy das Wintergfrist mit dem Schlaaf empfahen mögind. Der Eychhorn zücht ein breyte Schindlen oder Schyt mit dem Mul an'B Gstad und fart daruff über wie in einem Schyff, richtet den dicken Schwantz wie ein Sägeltủch uf; daryn gadt denn der Nachwind und trybt's, daß er keines andern Sägels bedarf. Wöliche Stymm, wöliche Red möchte die göttliche Wyßheit dermaß als die allerminsten und schlächtesten Tierlin ußkünden ? $\mathrm{J}_{a}$ ouch die Ding, die kein Befintnuß habend, die bezügend, daß die Macht, Güte und läbendmachende Krafft Gottes allweg inen gegenwürtig sye. Als so die Erden, die allen Dingen Narung gibt, sich alle Zyt erbitten laßt, daß sy gnůgsame und überflüssige Narung gibt, und gedenckt nit an die Wunden, die iren mit Houwen, Kersten, Mässeren und Sächen gestochen und gehouwen werdend. So der Touw, der Rägen und die Wasserflüß, alles das, das sunst vor Dürre nit wachsen mag, dermaa $ß$ uferweckend, füchtigend und erfristend, daß sy mit dem wunderbaren Wachsen und Zůnemmen der göttlichen Krafft und Läbens Gegenwürtigkeyt bezügend. Was sol ich von'n Bärgen sagen? Die doch ein gar grobe unkönnende Burde und Größe sind, so sy die Erden, glich wie das Gebeyn das Fleysch, verhaltend und bevestnend, so sy den Gang von einem Ort an das ander gar verhaltend oder gar kümmerlich zůlassend, und wiewol sy schwärer sind weder die Erden; noch schwymmend sy entbor und fallend nit zů Boden; kündend sy mit disem allen die unzerbrochne Macht Gottes und die Burde und Größe siner Maiestat nit uß ? Und darumb so findend wir in disen Dingen allen die Gegenwürtigkeit göttlicher Krafft, durch die sy sind, bstond, läbend und bewegt werdend, nit minder dann ouch im Menschen.»(ZH 2, S. 115f.) Zwingli staunt nur und schließt: "Wär möchte aber alle Gschlächte der Creaturen erzellen, so täglich etlich nüwe, die bißhar noch nye gesähen oder zum minsten vergessen sind, harfür kommend, daß von Anfang der Wält har nye kein Mensch aller Dingen Wüssen gnůgsam hat mögen haben? » (ZH 2, S. 140.)

Hinter dieser Freude Zwinglis an der Wunderbarkeit und Mannigfaltigkeit der Natur stand natürlich, als zweite seiner Grundanschauungen gewissermaßen, ein äußerst lebendiger Schöpfungsglaube. Zwingli war wie nicht zuletzt die wiederholte Durchführung des kosmologischen und physiko-teleologischen Gottesbeweises zeigt - felsenfest überzeugt, daß die Welt weder ewig bestanden hat noch irgendwie von selber geworden sei; Gott hat sie vielmehr aus dem Nichts geschaffen, und Gott erhält sie seither auch in einer fortwährenden Neuschöpfung. Wir wollen auch hier wieder auf den Reformator selber hören. Was die Schöpfung im 
eigentlichen Sinn betrifft, erinnern wir allerdings nur an die schon zitierten Worte über 1.Mose 1 der Schöpfung, an andere Worte über Schönheit und sagen zusammenfassend, wie Zwingli namentlich wieder in seiner Schrift «über die Vorsehung» betont, daß keine Kraft noch Macht außerhalb Gottes ist, Gott die Quelle ist, und wenn man so sagen darf, die Substanz, aus der alles ausfließt. Im übrigen möchte ich lieber auf ein paar Stellen hinweisen, in denen Zwingli von der creatio continua spricht. Zunächst allgemein, es heißt da etwa: Gott ist das «Sein aller Dinge, ihr Wesen und ihr Bestand»; "alles, was ist, ist Gott; denn er ist deswegen, weil Gott ist und sein Wesen ausmacht». Dementsprechend ist die Natur nichts anderes als die fortdauernde Wirkung Gottes », oder: «Die irren, welche in der Natur etwas anderes finden als die allezeit wirksame Kraft und Vorsehung Gottes $\aleph^{5}$. Wiederum in der Predigt «von der Vorsehung» sagt Zwingli unter Hinweis auf Zeugnisse 2. Mose 3, 14 (Ich bin der ich bin), Rö. 11, 36 (Aus ihm und durch ihn und in ihm sind alle Ding), Apg. 17, 24-29 (in ihm leben und weben und sind wir): «Damit wir nun uff das Vorig, so oben angehebt, widerumb kummind, sind die Gstirne, die wachsenden Pflantz, die Thier und andere Ding allein darumb, daß sy $u ß$ dem und $u ß$ dem sind, das allweg und allein ist (und das ist Gott), ja das also ist, daß, ob du dichtetest (das doch unmüglich ist), daß es nit wäre, von Stund an, so bald es nit wäre, nüt wäre noch syn möchte; ja wo Gott nit wäre, wurde von Stund an Gstirn, Erden, Meer, ja die gantze Wält in einem Ougenblick zerfallen und zenüte werden» (ZH 2, S. 111). Noch schöner, das eben gesagte ergänzend, ist indes eine Stelle in der sogenannten Credo-Predigt, da Zwingli zur Demut mahnend fragt: «Wannen sind wir? Oder wär sind wir? Oder worin sind wir? Sind wir von uns selbs? Worumb sind wir denn nit stercker, wyser, schöner? Sind wir aber von unsern Vätteren und Můteren? Worumb sind wir denn nit holdsäliger, rycher, herlicher und größer? So doch kein Zwyffel ist, wenn wir von uns selbs oder von unseren Vätteren, wir wärind zum höchsten wys, starck, schön etc. Ja, wir langtend durch den Himmel hinuff und überlangtend Gott. Wer sind wir aber? Sind wir Schöpfer oder Geschöpfte? Wir sind on Zwyfel geschaffen und nit von uns selbs, wie erst gehört ist; warumb gebend wir uns dann etwas zů? So wir doch erkennend, daß unser Schöpfer allein der ist, der alle Ding erschaffen hatt. Worin sind wir aber? Sind wir in uns selbs, worumb erhaltend wir uns dann nit, daß wir nit altind, siechind oder sterbind? Daruß wir ring ermessend: so uns weder Sonn

5 Zitate nach Rudolf Stäehelin, Huldrejch Zwingli, sein Leben und Wirken, Bd. 2, Basel 1897, S. 186. 
noch Mon, Lufft noch Werme Frücht noch Kelte geben mögend, daß wir inn Wäsen unabgenglich blybind, ouch wir uns selbs das nit geben mögend noch unsere größten Fründ, daß wir keinen Athemzug thünd, one daß Gott unsere Krafft, die dem Atem zücht, und der Athem ouch im Athem ist. Warumb halt denn der Mensch so vil uf sich selbs? 》 (ZH 2, S. 27.) Es ist allein Gottes Liebe, die alles und auch uns erhält.

In diesem Zusammenhang stellen sich nun allerdings noch eine Reihe von Fragen. Wir müssen uns erstens einmal fragen: wenn Gott so alles in allem wirkt, wenn er es ist, der in den Gestirnen leuchtet und die Saat aufsprossen läßt, wenn er wesentlich gegenwärtig ist in allen Dingen, alles in Gott beschlossen ist, ist das eigentlich Pantheismus? Diese Frage ist rasch in negativem Sinn erledigt, wenn wir beachten, daß das «Sein Gottes in den Dingen nicht als Naturnotwendigkeit aufgefaßt wird, sondern als Gottes freie schöpferische Tätigkeit (Stähelin a.a.O., S. 187), als Ausdruck seiner frei sich mitteilenden Gnade und Liebe. Die Dinge, Geschöpfe der Natur sind zwar aus Gott und in Gott, sie sind Gottes Wesen, Kraft und Wirkung, sie sind als solche aber doch nichts als Mittel, Geschirre und Werkzeuge, durch die die göttliche Kraft wirkt und nicht Ursachen, denn die alleinige Ursache ist Gott. «Und daß wir's in einer Summ sagind, so redend wir also: Die Erden bringt nit Frucht, das Wasser befůchtet und neert nit, der Lufft macht nit fruchtbar, das Fhür wärmt nit, ja die Sonnen nit; sunder die Krafft, die ein Ursprung, Läben und Stercke ist, aller Dingen, brucht die Erden als ein Gschirr zủ gebären und Frucht ze bringen. Diewyl dann die Ding, die harfürbracht sind, mit Spyß erhalten werdend, spyßt und tränckt sy Gott durch das Wasser; darnach erkůlt und erfristet er sy durch den Lufft, füllt sy und meeret sy, und denn brütet er's durch das Fhür und Sonnen us, macht's sytig, schön und hüpsch, fürderet's und macht's zů vollen uß.» Und im gleichen Zusammenhang, etwas weiter unten: «Das merck also: Wenn wir sähend, daß die Erden Frucht gibt, die Böum Ops bringend, die Sonnen iren Schyn und Hitz ußgüßt, so söllend wir trachten und warnemmen, daß Gott uns syn Hand bütet, glych als so ein früntlicher Vatter synen lieben Kindlin einen Truben an einem Räbschoß bütet. Es ist die gůtige, früntliche Krafft Gotts, die uns alle Ding gibt: die Erden aber, die Böum, die Sonnen und alle anderen Ding sind das Schoß und der Truben, an dem uns die Gaab geben wirt und an dem wir's nemmen und empfahend» (ZH 2, S 127f.). Es ist in diesem Zusammenhang auch zu denken an eine Stelle in der Psalmenauslegung, da Zwingli zu Psalm 111 sagt: «Infidelis (d.h. der Ungläubige): Die natur ist ein abgöttin denen, die alle Ding der natur zuogebind. Fidelis (Gläu- 
bige) : aber der glöubig gibts allein gott zuo, der die natur geschaffen hat; das thuot der, der Gott erkennt ${ }^{6} . »$

Wir erkennen gerade diesen Sachverhalt noch deutlicher, wenn wir zweitens uns nun der Frage zuwenden, wie Zwingli über den zu seiner Zeit verbreitetsten $Z$ weig der Naturforschung gedacht hat, über die Astronomie und deren unglückliches Kind, die Astrologie. Das ganze 16. Jahrhundert glaubte bekanntlich noch daran, selbst Kopernikus und Kepler hielten an ihr fest, und von Melanchthon spottete Luther ja, er treibe Astrologie «wie ich einen starken Trunk Biers trinke, wenn ich schwere Gedanken habe" (Tischr. 1, 17). Wie hielt es Zwingli ? Wieder in der Schrift «über die Vorsehung» gibt der Reformator selber ein Beispiel aus dem Jahr 1524. In diesem Jahr drohten die Sterngucker große Wassergüsse, Verderben aller Dinge an. Gleichzeitig schlugen die Theologen Lärm, das Ende der Welt wäre da. Dazu sagt Zwingli wörtlich: «Die Sternensäher habend sölichs uß Zemenfügung und Zwytracht des Gstirns ermässen und wargenommen; die Predicanten aber habend sölichs uß Gewüsse des Gloubens, uß Gottlose und Verlesterung, uß Zerstörung aller Erberkeyt und gůter Sitten ersähen; dann wo dise Ding under den Menschen überhand nämmend, mögend keine Sternensäher uß ihrer Kunst der Thüre (Teuerung) und Wölfele (wohlfeile Zeit) so gwyß syn, als die Propheten gwiß sind, daß die göttliche Raach und Straaff vorhanden sye. Das Jar vergieng also, daß des Wassers halb noch Platz was, uff Erden ze wonen. Und ob glych an ettlichen Orten die Wasser groß wurdend, sind doch in Eidgnossen in vil Jaren die Wasser nie kleiner gsin, wie wol darneben ze besorgen (befürchten war), daB der Huff aller Lastren, Undertruckung und Felschung gmeyner Gerechtigkeyt und die Unverschampte aller Boßheyt und Schalkheyt ein vil schädlicher Wasserguß (dann wenn die Lyb der Menschen und des Vychs ertrunckend) bringen werde, wöliches die Propheten vil waarer dann die Sternensäher vorgeseyt habend, ob glych yetweder den andren darzwüschen gar styff verachtet, so sy dargegen billicher uff einander mit Eersamkeyt achthaben soltend. Der Sternensäher, so er sähe, daß die Gstirn etwas Ruchs tröwtend, solte er darneben der Wyßheit Gottes mit Verwundernuß warnemmen, daß sy die oberen Ding von der Zyt, als der Hymmel geschaffen ist, so ordenlich gstelt hette, daß sy sölich Ungewitter gerad uff die Zyt tröwtend, uff die der Menschen Boßheyt und Verlesterung aller Dingen zum höchsten wäre. Die Propheten aber, ob sy glych das unmäßig und frävel Vorsagen der Sternensäher billich

${ }^{6}$ Zitiert nach Gottfried W. Locher, Die Theologie Huldrych Zwinglis im Lichte seiner Christologie, Zürich 1952, S. 87. Vgl. dort auch S. 94f., wo Locher näher auf die Frage des Pantheismus eingeht. 
schältend und verwerffend, soltend sy, die doch die waare und rechte Kunst der Astronomy, das ist: des Gstirns UBrechnung und Louff, als wenig verachten als das Gstirn selbs. Dann wie das Gstirn ein Mittel und Werchzüg ist, durch wölches die göttliche Krafft sich öugt und ußgüßt, also ist ouch das Wüssen ires Louffs und irer Ordnung nüt anders dann ein Wüssen der göttlichen Würckung. Deßhalb myns Bedunckens die Propheten keyn Kunst minder verachten söllend weder die Astronomy. So kumpt nun der Uffrůr und Entbörung, so yetz in der Wält ist, nit one Gfärd, wielands oder uß Zorn des Gstirns, sunder uß göttlicher Fürsichtigkeyt, die alle Ding von Anfang also geordnet und gesetzt hatt, daß die Propheten den göttlichen Zorn und Raach tröuwen und die Sternensäher künfftige Übel und Schaden vorsagen möchtind, und die beden Ding werdend $u ß$ der Güte Gottes uns anzeigt, damit uns der Wäg zur Besserung und zum Heyl dester mee offen stande» (ZH 2, S. 211 ff.). Wenn diese Lösung des Problems unserm heutigen Denken bestimmt auch nicht mehr entspricht, kann man Zwingli die Bewunderung nicht versagen: einerseits stimmt er mit der allgemeinen Ansicht seiner Zeit überein, daß Kometen, Regenbogen und andere Himmelserscheinungen, Sonnen- und Mondfinsternisse, selbstverständlich auch Erdbeben (die nach Zwingli übrigens auf Grund unterirdischer Winde entstehen; ZH 2, S. 103 oben) ein Fingerzeig Gottes sein könnten. Anderseits aber erscheint es ihm doch gefährlich, nur darauf abzustellen. Das Geschick der Menschen, das Schicksal der Welt liegt letztlich nicht in den Sternen, sondern in Gottes Hand. Gott hat es vorbestimmt, und diese Vorbestimmung zu erkennen, zu deuten ist dann auch in erster Linie Aufgabe der Theologen! In einer Predigt über Jeremia 10 warnt Zwingli denn auch nachdrücklich vor jedem Mißbrauch der Zeichen des Himmels! Die Gestirne sind eigentlich da «um Tag und Nacht einzuteilen, daß Zeichen und Zeiten seien und überhaupt auch, um das fruchtbare Wachstum zu bewirken, daß man Zeit und Zeichen wisse zu unserm Nutzen, wie zum Beispiel um den Acker anzubauen, um zu schneiden und zu heuen... Sie haben auch keine Vernunft, sie stehen darum nicht über der menschlichen Seele. Diese kann nur unter die rechte Kraft Gottes gezwungen werden, der auch Sonne und Mond und alle Gestirne braucht und mit ihnen arbeitet wie mit seinem Werkzeug, ja mit seinem Handgeschirr» (Zwingli Predigten, S. 197f.). Es mag damit in einem gewissen Widerspruch stehen, wenn Zwingli einmal dann doch mit Dürers «Melancholie» psychologische Erkrankungen auf eine «saturnische, melancholische Eigenrichtigkeit und Lätzköpfige» zurückführt (Z IV, S. 287 f., s. dort auch Anm. 13) und auch die Täufer als solche «Saturn-Kinder» charakterisiert (Z III, S. 
405) - in beiden Fällen allerdings ohne genauere theologische Begründung.

Die dritte Frage betrifft schließlich den Platz, den für Zwingli das Wunder in der Natur einnimmt. Wunder sind wie die eben genannten Naturerscheinungen am Himmel von der Vorsehung vor der Erschaffung der Welt in ihren Plan aufgenommen und dem Naturzusammenhang eingefügt worden. Sie sind jedoch eher da für die Schwachen als für die fest im Glauben stehenden; paradoxerweise sind nicht Wunder das eigentlich Wunderbare, sondern die einmal fest und unveränderlichen Ordnungen der Natur. Ja, sagt Zwingli, «Die Ding, die nach gemeynem Louff des Gestirns und der Natur on nüwe Wunderwerck gschähend, sind vil wunderbarer (deßhalb daß die Ordnung so styff und unverruckt für und für bstaat) dann die Ding, die uff einmal allein neüwerwyß nach Gelägenheit der Sach gschähend» (ZH 2, S. 211). Das heißt nun allerdings nicht, daß Gott eben nicht dann und wann eingreifen könnte, Gott steht auch über diesen Ordnungen. Wie Zwingli in seiner Marienpredigt meint, ist der Lauf der Natur nicht im Stande, den Schöpfer und Ordner aller Dinge zu zwingen, daß er sein Wirken nach ihnen richten müsse. Die Natur, die ihren Gang und ihre Gewohnheit von Gott hat, muß sich umgekehrt von Gott zwingen und führen lassen. "Auch leidet sie nichts Unbilliges, wenn ihr Lauf gehindert oder geändert wird, nicht mehr als wenn ein beliebiger Arbeiter in einem Haushalt vom Hausvater geheißen wird seine Arbeit anders und geschickter zu verrichten. Das heißt bei uns ein miraculum, das ist: Wunder. Aber an sich, das heißt nach dem Wirken Gottes, ist es kein Wunder. Denn Gott ist nichts unmöglich. In seiner Hand stehen alle Dinge, mit diesen kann er schaffen und gebieten wie er will; keiner darf, wie Paulus Rö. 9, 20 sagt, sprechen: ,Warum hast du mich so gemacht?"» (ZH 1, S. 148f.)

Wir kommen zum Schluß. Nehmen wir alle Grundgedanken $Z$ winglis über die Natur, über das Verhältnis der Natur zu Gott zusammen, so stellen wir in der Hauptsache zwei Dinge fest: einmal sah der klare Blick unsres deutschschweizerischen Reformators im Großen und Kleinen, in den Dingen des Makrokosmos so gut wie in einem Rebblatt das Wunder, welches die Natur bildet, er konnte staunen, bewundern, preisen. Zum zweiten besaß Zwingli einen tiefen Sinn dafür, daß dieses Wunder letztlich ein Werk Gottes ist. Auch er könnte so, wie Calvin in der Institutio (I 5, 5) gesagt haben: «Wenn man es nur recht versteht, so kann von einem gläubigen Gemüt gesagt werden, die Natur sei Gott.» Von dieser Behauptung aus müssen wir uns freilich noch ganz kurz fragen, ob Zwingli nicht notwendigerweise «natürliche Theologie» getrieben habe. Ohne Zweifel ist - wie übrigens auch seinen Mitreformatoren Luther und 
Calvin - seiner Meinung nach der Natur eine gewisse Offenbarungsqualität eigen. Unter Berufung auf den Locus classicus aller natürlichen Theologie, Rö. 1, 20 kann $Z$ wingli verschiedentlich davon reden, daß die Betrachtung der äußern Dinge ein Weg zur Erkenntnis Gottes ist: den Gläubigen zum Guten, «den Ungläubigen aber zur Bezeugung ihrer verdienten Verdammnis» (Predigt über Jes. 40; S. 110). Ein wesentlicher Weg zur Gotteserkenntnis ist das aber doch nicht. Wenn Zwingli auch, wie wir gehört haben, nicht etwa nur dem Menschen, sondern aller Kreatur zubilligt, daß sie Gottes Ehre und Gottes Macht verkündige, sagt er im entscheidenden Kapitel seines Commentarius «Von Gott» doch, daß letztlich der Glauben an Gott nur durch die Kraft und Gnade dessen geschieht, an den man glaubt. «Es ist offenbar, daß die Frommen darum glauben, daß es einen Gott gebe und daß die Welt sein Werk sei usw., weil sie es von Gott gelehrt sind. Also ist es einzig Gottes Werk, sowohl, daß du glaubst, daß ein Gott sei, als auch, daß du diesem Gott vertraust» (ZH 9, S. 23). Und nun mit einem Vergleich zu schließen: «Was aber Gott sei, das wissen wir aus uns ebensowenig, wie ein Käfer weiß, was der Mensch ist. Ja, das unendliche und ewige Göttliche hat vom Menschen einen viel größern Abstand als der Mensch vom Käfer, weil ein Vergleich der Geschöpfe untereinander eher angebracht ist als der Vergleich der Geschöpfe mit dem Schöpfer. Und alle vergänglichen Dinge sind sich gegenseitig viel näher und verwandter, als sie dem ewigen unbegrenzten Göttlichen sind, obschon man in den Geschöpfen Abbilder und Fußspuren, wie man sagt, des Göttlichen findet...» (ZH 9, S. 23.) Wir kommen aus eigener Kraft nicht zur Erkenntnis Gottes; was Gott ist, können wir nur aus seinem Mund erlernen: durch Christus.

Diesen Aufsatz widmet der Verfasser in Dankbarkeit Herrn Professor D. Fritz Blanke zum 60. Geburtstag 